\title{
Parametric Instability of Woven Fiber Laminated Composite Plates in Adverse Hygrothermal Environment
}

\author{
M. K. Rath*, M. K. Dash \\ Department of Civil Engineering, IGIT, Sarang, Odisha (INDIA) \\ *Corresponding author: manojrat@yahoo.com
}

Received May 20, 2014; Revised June 04, 2014; Accepted June 16, 2014

\begin{abstract}
The present paper deals with the parametric instability of woven fiber laminated composite plates subjected to in plane periodic loadings in hygrothermal environment. The effects of various parameters like increase in number of layers, aspect ratios, side-to thickness ratios, static load factors, ply-orientations, lamination angle and the degree of orthotropy of simply supported composite plates at elevated temperatures and moisture concentrations on the principal instability regions are investigated using finite element method using Bolotin's approach. The effects of transverse shear deformation and rotary inertia are used to study the anti-symmetric angle-ply rectangular plates. The onset of instability occurs earlier and the width of dynamic instability regions increases with rise in temperature and moisture for different parameters.
\end{abstract}

Keywords: parametric instability, static load factor, orthotropic, angle-ply, hygrothermal environment, lamination sequence

Cite This Article: M. K. Rath, and M. K. Dash, "Parametric Instability of Woven Fiber Laminated Composite Plates in Adverse Hygrothermal Environment.” American Journal of Mechanical Engineering, vol. 2, no. 3 (2014): 70-81. doi: 10.12691/ajme-2-3-4.

\section{Introduction}

Fiber-reinforced composite materials are extensively used in thin walled structural components of various modern engineering structures in the high performance application areas, aerospace, submarines, automobiles and other application areas in which it reduce the overall operational cost have necessitated a strong need to understand their dynamic characteristics under different loading conditions. The composite plate structures are sometimes subjected to periodic in-plane load and become dynamically unstable for certain combinations of load amplitude and disturbing frequency. The above phenomenon is called parametric instability or parametric resonance of elastic structures. Several means of combating parametric resonance such as damping and vibration isolation may be inadequate and sometimes dangerous with reverse results. A number of catastrophic incidents can be traced to parametric resonance. In contrast to the principal resonance, the parametric instability may arise not merely at a single excitation frequency but even for small excitation amplitudes and combination of frequencies. Thus the parametric instability characteristics of laminated composite plates subjected to hygrothermal loads are of great technical importance for understanding the dynamic system under harmonic loadings.

Sai Ram and Sinha [1] investigated the effects of moisture and temperature on the free vibration of laminated composite plates using finite element method. Chen and Chou [2] studied the free vibration analysis of orthogonal-woven fabric composites using onedimensional elasto-dynamic analysis. Shen et al [3] discussed the dynamic response of shear deformable laminated plates in hygrothermal environments based on a micro-mechanical model. Fakhari and Ohadi [4] studied the large amplitude vibration of functional graded material (FGM) plates under thermal gradient and transverse mechanical loads using finite element method. However, the static stability studies are less available in literature. Dewey and Costello [5] studied the thermal buckling of non-homogeneous plates using the second variation of the potential energy.

Sai Ram and Sinha [6] investigated the effects of moisture and temperature on the static stability of laminated composite plates. The mathematical model based on finite element method which takes transverse shear deformation into account. Jones [7] proposed the thermal buckling of uniformly heated unidirectional and symmetric cross-ply laminated fiber reinforced composites uniaxial in-plane restrained simply supported rectangular plates. Dash et al. [8] presented an experimental study on the effects of corrosion on elastic buckling and post buckling response of unidirectional Eglass/epoxy composite rectangular plates subjected to compressive load and liquid environment exposure.

Few literatures are available on dynamic instability of unidirectional composite plates under ambient temperature and moisture subjected to periodic in-plane loads. Bert 
and Birman [9] studied the dynamic instability of shear deformable anti-symmetric angle-ply plates by using finite element method. Moorty et al. [10] presented the parametric instability of laminated composite shear deformable flat panels subjected to in plane edge loads using finite element method. Cheng-ti [11] observed the theory of nonlinear dynamic stability of composite laminated plates using Hamilton principle. Mond and Cederbaum [12] presented the dynamic stability of antisymmetric laminated plates by using the method of multiple scales. Liao and Cheng [13] observed the dynamic instability of stiffened laminated composite plates subjected to pulsating forces using finite element equation of motion. Ganapathi [14] proposed the dynamic instability of laminated composite plates subjected to thermal loads using first order shear deformation theory and Lagrange's equation. Radu [15] studied the dynamic instability of composite laminates using a higher order theory. The procedure implemented by using the finite element approach. Sahu and Dutta [16] investigated the dynamic instability of laminated composite rectangular plates subjected to non-uniform harmonic in-plane edge loading using finite element methods. Wang and Dawe [17] proposed the dynamic instability of composite laminated rectangular plates by using Lagrange's formulation. $\mathrm{Wu}$ and Shih [18] presented the dynamic stability of rectangular plates with an edge crack by applying Galerkin's method. Wu and Shih [19] studied the dynamic instability of arbitrarily laminated skew plates based on Von Karmans plate theory, the large amplitude dynamic equation of thin laminated plates are derived by applying double Fourier series. Liew et al. [20] investigated the dynamic stability analysis of thin laminated cylindrical panels under static and periodic axial forces by using the mesh-free Ritz method. Lanhe et al. [21] studied the dynamic stability analysis of FGM plates by the moving least squares differential quadrature method. Chen et al. [22] studied the dynamic stability of laminated hybrid composite plates subjected to periodic uniaxial and bending stress and the instability region is marked by Bolotin's method. Fazilati and Ovesy [23] presented the dynamic instability analysis of composite laminated thin walled structures using finite strip method. However, in most of the above-mentioned studies on dynamic instability of unidirectional composite plates in ambient environment are reported. To the best of author's knowledge, no work is reported in literature on parametric instability of industry driven woven fiber laminated composite plates subjected to hygrothermal environment. Due to its practical importance and uniqueness in the above fields, this paper discuss the effects of static load factors, ply-orientations, lamination angle, orthotropic on the parametric resonance characteristics of laminated composite plates under higher temperature and moisture environments.

\section{Mathematical Formulation}

The mathematical formulation for parametric instability behavior of laminated composite plates subjected to moisture and temperature are presented. Consider a laminated plate of uniform thickness ' $t$ ' consisting of a number of thin laminae, each of which may be arbitrarily oriented at an angle ' $\theta$ ' with reference to the X-axis of the co-ordinate system as shown in Figure 1 and Figure 2.

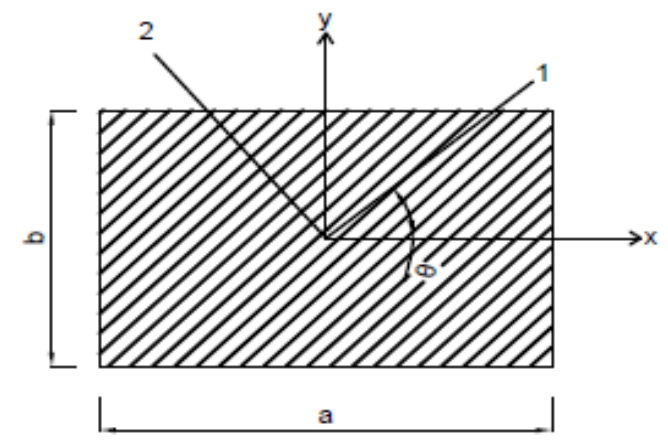

Figure 1. Arbitrarily oriented laminated plate

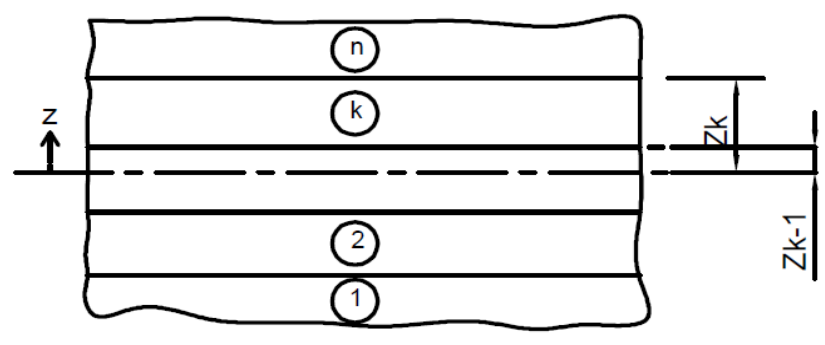

Figure 2. Geometry of an n-layered laminate

\subsection{Governing Equations}

The governing differential equations for vibration of a shear deformable general laminated composite plates and shells in general are specified here but the scope of the analysis is for composite plates. The behavior of laminated composite plates in hygrothermal environment derived on the basis of first order shear deformation theory subjected to in-plane loads are;

$$
\begin{aligned}
& \frac{\partial N_{x}}{\partial x}+\frac{\partial N_{x y}}{\partial y}+\frac{Q_{x}}{R_{x}}+\frac{Q_{y}}{R_{x y}}=P_{1} \frac{\partial^{2} u}{\partial t^{2}}+P_{2} \frac{\partial^{2} \theta_{x}}{\partial t^{2}} \\
& \frac{\partial N_{x y}}{\partial x}+\frac{\partial N_{y}}{\partial y}+\frac{Q_{y}}{R_{y}}+\frac{Q_{x}}{R_{x y}}=P_{1} \frac{\partial^{2} v}{\partial t^{2}}+P_{2} \frac{\partial^{2} \theta_{y}}{\partial t^{2}} \\
& \frac{\partial Q_{x}}{\partial x}+\frac{\partial Q_{y}}{\partial y}-\frac{N_{x}}{R_{x}}-\frac{N_{y}}{R_{y}}-2 \frac{N_{x y}}{R_{x y}} \\
& +N_{x}^{a} \frac{\partial^{2} w}{\partial x^{2}}+N_{y}^{a} \frac{\partial^{2} w}{\partial y^{2}}+N^{a}{ }_{x y} \frac{\partial^{2} w}{\partial x \partial y}=P_{1} \frac{\partial^{2} w}{\partial t^{2}} \\
& \frac{\partial M_{x}}{\partial x}+\frac{\partial M_{x y}}{\partial y}-Q_{x}=P_{3} \frac{\partial^{2} \theta_{x}}{\partial t^{2}}+P_{2} \frac{\partial^{2} u}{\partial t^{2}} \\
& \frac{\partial M_{x y}}{\partial x}+\frac{\partial M_{y}}{\partial y}-Q_{y}=P_{3} \frac{\partial^{2} \theta_{y}}{\partial t^{2}}+P_{2} \frac{\partial^{2} v}{\partial t^{2}}
\end{aligned}
$$

Where $\mathrm{N}_{\mathrm{x}}, \mathrm{N}_{\mathrm{y}}$ and $\mathrm{N}_{\mathrm{xy}}$ are the in-plane stress resultants, $\mathrm{M}_{\mathrm{x}}, \mathrm{M}_{\mathrm{y}}$ and $\mathrm{M}_{\mathrm{xy}}$ are moment resultants and $\mathrm{Q}_{\mathrm{x}}, \mathrm{Q}_{\mathrm{y}}=$ transverse shear stress resultants. $\mathrm{R}_{\mathrm{x}}, \mathrm{R}_{\mathrm{y}}$ and $\mathrm{R}_{\mathrm{xy}}$ identify the radii of curvatures in the $\mathrm{x}$ and $\mathrm{y}$ direction and radius of twist.

$$
\left(P_{1}, P_{2}, P_{3}\right)=\sum_{k=1}^{n} \int_{z_{k-1}}^{z_{k}}(\rho)_{k}\left(1, z, z^{2}\right) d z
$$


Where $n=$ number of layers of laminated composite twisted curved panel, $(\rho)_{\mathrm{k}}=$ mass density of $\mathrm{k}_{\mathrm{th}}$ layer from mid-plane.

\subsection{Dynamic stability studies}

The equation of motion for vibration of a laminated composite panel in hygrothermal environment, subjected to generalized in-plane load. $N(t)$ May be expressed in the matrix form as:

$$
[M]\{\ddot{q}\}+\left[\left[K_{e}\right]-N(t)\left[K_{g}\right]\right]\{q\}=0
$$

' $q$ ' is the vector of degrees of freedoms $\left(u, v, w, \theta_{x}, \theta_{y}\right)$. The in-plane load ' $\mathrm{N}(\mathrm{t})$ ' may be harmonic and can be expressed in the form:

$$
N(t)=N_{s}+N_{t} \operatorname{Cos} \Omega t
$$

Where $N_{s}$ the static portion of load $\mathrm{N}$ (t), $N_{t}$ the amplitude of the dynamic portion of $N(t)$ and $\Omega$ is the frequency of the excitation. The stress distribution in the panel may be periodic. Considering the static and dynamic component of load as a function of the critical load,

$$
N_{s}=\alpha N_{c r}, N_{t}=\beta N_{c r}
$$

Where $\alpha$ and $\beta$ are the static and dynamic load factors respectively. Using Eq. (5), the equation of motion for panel in hygrothermal environment under periodic loads in matrix form may be obtained as:

$$
\begin{aligned}
& {[M]\{\ddot{q}\}+\left[\left[K_{e}\right]-\alpha N_{c r}\left[K_{g}\right]\right.} \\
& \left.-\beta N_{c r}\left[K_{g}\right] \operatorname{Cos} \Omega t\right]\{q\}=0
\end{aligned}
$$

The above Eq. (6) represents a system of differential equations with periodic coefficients of the Mathieu-Hill type. The development of regions of instability arises from Floquet's theory which establishes the existence of periodic solutions of periods $\mathrm{T}$ and $2 \mathrm{~T}$. The boundaries of the primary instability regions with period $2 \mathrm{~T}$, where $\mathrm{T}=2$ $\pi / \Omega$ are of practical importance and the solution can be achieved in the form of the trigonometric series:

$$
q(t)=\sum_{k=1,3,5, . .}^{\infty}\left[\left\{a_{k}\right\} \operatorname{Sin}(k \Omega t / 2)+\left\{b_{k}\right\} \operatorname{Cos}(k \Omega t / 2)\right]
$$

Putting this in Eq. (6) and if only first term of the series is considered, equating coefficients of $\operatorname{Sin} \Omega \mathrm{t} / 2$ and $\operatorname{Cos}$ $\Omega \mathrm{t} / 2$, the equation (6) reduces to

$$
\begin{aligned}
& {\left[\left[K_{e}\right]-\alpha P_{c r}\left[K_{g}\right]\right.} \\
& \left. \pm \frac{1}{2} \beta P_{c r}\left[K_{g}\right]-\frac{\Omega^{2}}{4}[M]\right]\{q\}=0
\end{aligned}
$$

Eq. (8) represents an eigenvalue problem for known values of $\alpha, \beta$ and $P_{c r}$. The two conditions under the plus and minus sign correspond to two boundaries (upper and lower) of the dynamic instability region. The above eigenvalue solution give of $\Omega$, which give the boundary frequencies of the instability regions for the given values of $\alpha$ and $\beta$. In this analysis, the computed static buckling load of the panel is considered as the reference load. Before solving the above equations, the stiffness matrix $[\mathrm{K}]$ is modified through imposition of boundary conditions.

\subsection{Constitutive Relation}

The Constitutive relations for the plate subjected to moisture and temperature are:

$$
\{F\}=[D]\{\varepsilon\}-\left\{F^{N}\right\}
$$

Where

$$
\begin{aligned}
& \{F\}=\left\{N_{x}, N_{y}, N_{x y}, M_{x}, M_{y}, M_{x y}, Q_{x}, Q_{y}\right\}^{T} \\
& \left\{F^{N}\right\}=\left\{N_{x}{ }^{N}, N_{y}{ }^{N}, N_{x y}, M_{x}{ }^{N}, M_{y}{ }^{N}, M_{x y}{ }^{N}, 0,0\right\}^{T} \\
& \{\varepsilon\}=\left\{\varepsilon_{x}, \varepsilon_{y}, \gamma_{x y}, K_{x}, K_{y}, K_{x y}, \phi_{x}, \phi_{y}\right\}^{T}
\end{aligned}
$$

Where, $N_{x}, N_{y}, N_{x y}=$ in-plane internal stress resultants.

$M_{X}, M_{y}, M_{x y}=$ internal moment resultants.

$Q_{X}, Q_{y}=$ transverse shear resultants.

$N_{x}{ }^{N}, N_{y}{ }^{N}, N_{x y}{ }^{N}=$ in-plane non-mechanical stress resultants due to moisture and temperature.

$M_{x}{ }^{N}, M_{y}{ }^{N}, M_{x y}{ }^{N}=$ non- mechanical moment resultants due to moisture and temperature.

$\varepsilon_{x}, \varepsilon_{y}, \gamma_{x y}=$ in-plane strains of the mid-plane.

$K_{X}, K_{Y}, K_{x y}=$ Curvature of the plate

$\phi_{x}, \varphi_{y}=$ Shear rotations in $\mathrm{x}-\mathrm{z}$ and $\mathrm{y}-\mathrm{z}$ planes, respectively.

$$
[D]=\left[\begin{array}{cccccccc}
A_{11} & A_{12} & A_{16} & B_{11} & B_{12} & B_{16} & 0 & 0 \\
A_{12} & A_{22} & A_{26} & B_{12} & B_{22} & B_{26} & 0 & 0 \\
A_{16} & A_{26} & A_{66} & B_{16} & B_{26} & B_{66} & 0 & 0 \\
B_{11} & B_{12} & B_{16} & D_{11} & D_{12} & D_{16} & 0 & 0 \\
B_{12} & B_{22} & B_{26} & D_{12} & D_{22} & D_{26} & 0 & 0 \\
B_{16} & B_{26} & B_{66} & D_{16} & D_{26} & D_{66} & 0 & 0 \\
0 & 0 & 0 & 0 & 0 & 0 & S_{44} & S_{45} \\
0 & 0 & 0 & 0 & 0 & 0 & S_{45} & S_{55}
\end{array}\right]
$$

The non-mechanical force and moment resultants due to moisture and temperature are expressed as follows.

$$
\begin{aligned}
& \left.\qquad N_{x}{ }^{N}, N_{y}{ }^{N}, N_{x y}{ }^{N}\right\}^{T}=\sum_{K=1}^{n}\left(\overline{Q_{i j}}\right)\{e\}_{k}\left(z_{k}-z_{k-1}\right) \\
& \quad \text { for } i, j=1,2,6 \\
& \left\{M_{x}{ }^{N}, M_{y}{ }^{N}, M_{x y}{ }^{N}\right\}^{T}=\frac{1}{2} \sum_{K=1}^{n}\left(\overline{Q_{i j}}\right)\{e\}_{k}\left(z_{k}{ }^{2}-z_{k-1}{ }^{2}\right) \\
& \text { for } i, j=1,2,6 \\
& \text { Where, } \\
& \{\varepsilon\}_{N}=\left\{\varepsilon_{x N}, \varepsilon_{y N}, \varepsilon_{x y N}\right\}^{T}=[T]\left\{\beta_{1} \beta_{2}\right\}_{k}{ }^{T}\left(C-C_{O}\right) \\
& +[T]\left\{\alpha_{1} \alpha_{2}\right\}_{k}{ }^{T}\left(T-T_{O}\right)
\end{aligned}
$$

In which, $[T]=$ Transformation matrix due to moisture and temperature and is given as: 


$$
[T]=\left[\begin{array}{cc}
\cos ^{2} \theta & \sin ^{2} \theta \\
\sin ^{2} \theta & \cos ^{2} \theta \\
\sin 2 \theta & \cos 2 \theta
\end{array}\right]
$$

$\varepsilon_{x N}, \varepsilon_{y N}, \varepsilon_{x y N}=$ non-mechanical strains due to moisture and temperature

$\beta_{1}, \beta_{2}=$ moisture coefficient along 1 and 2 axes of lamina, respectively

$\alpha_{1} \cdot \alpha_{2}=$ thermal coefficients along 1 and 2 axes of lamina, respectively

$T, T_{O}=$ Elevated and reference moisture concentration The stiffness coefficient is defined as:

$$
\begin{gathered}
\left(A_{i j}, B_{i j}, D_{i j}\right)=\sum_{k=1}^{n} \int_{z_{k-1}}^{k}\left[Q_{i j}\right]_{k}\left(1, z, z^{2}\right) \mathrm{dz}(\mathrm{i}, \mathrm{j}=1.2 .6)(13) \\
S_{i j}=\kappa \sum_{k=1}^{n} \int_{z_{k-1}}^{k}\left[Q_{i j}\right]_{k} d z(\mathrm{i}, \mathrm{j}=4,5)
\end{gathered}
$$

$\kappa=$ shear correction factor

$A_{i j}, B_{i j}, D_{i j}$ are the extensional, bending-stretching coupling and shear bending stiffnessess respectively. First order shear deformation theory (FSDT) is employed and a shear correction factor of 5/6 is included in $S_{i j}$ for all the numerical computations.

$\left(\overline{Q_{i j}}\right)$

$$
\begin{aligned}
& \mathrm{k} \text { in equations } 11 \text { and } 12 \text { is defined as: } \\
& {\left[\overline{\mathrm{Q}}_{\mathrm{ij}}\right]_{k}=\left[T_{1}\right]^{-1}\left[Q_{i j}\right]_{k}\left[T_{1}\right]^{-T}(\mathrm{i}, \mathrm{j}=1.2 .6)} \\
& {\left[\bar{Q}_{i j}\right]_{k}=\left[T_{2}\right]^{-1}\left[Q_{i j}\right]_{k}\left[T_{2}\right](\mathrm{i}, \mathrm{j}=4.5)}
\end{aligned}
$$

Where

$$
\begin{aligned}
& {\left[T_{1}\right]=\left[\begin{array}{ccc}
\cos ^{2} \theta & \sin ^{2} \theta & \sin \theta \cos \theta \\
\sin ^{2} \theta & \cos ^{2} \theta & -\sin \theta \cos \theta \\
-2 \sin \theta \cos \theta & 2 \sin \theta \cos \theta & \cos ^{2} \theta-\sin ^{2} \theta
\end{array}\right]} \\
& {\left[T_{2}\right]=\left[\begin{array}{cc}
\cos \theta & \sin \theta \\
-\sin \theta & \cos \theta
\end{array}\right]} \\
& {\left[Q_{i j}\right]_{k}=\left[\begin{array}{ccc}
Q_{11} & Q_{12} & 0 \\
Q_{12} & Q_{22} & 0 \\
0 & 0 & Q_{66}
\end{array}\right](\mathrm{i}, \mathrm{j}=1.2 .6)} \\
& {\left[Q_{i j}\right]_{k}=\left[\begin{array}{cc}
Q_{44} & 0 \\
0 & Q_{55}
\end{array}\right](\mathrm{i}, \mathrm{j}=4.5)}
\end{aligned}
$$

In which

$$
\begin{aligned}
& Q_{11}=\frac{E_{11}}{\left(1-v_{12} v_{21}\right)}, Q_{12}=\frac{E_{11} v_{21}}{\left(1-v_{12} v_{21}\right)}, \\
& Q_{21}=\frac{E_{22} v_{12}}{\left(1-v_{12} v_{21}\right)}, Q_{22}=\frac{E_{22}}{\left(1-v_{12} v_{21}\right)}, Q_{66}=G_{12}
\end{aligned}
$$

$E_{11}, E_{22}=$ Young's moduli of a lamina along and across the fibers, respectively.

$G_{12}, G_{13}, G_{23}=$ Shear moduli of a lamina with respect to 1,2 and 3 axes.

$v_{12}, v_{21}=$ Poisson's ratios.
The linear strains are defined as follows

The off-axis stiffness values are:

$$
\begin{aligned}
& \bar{Q}_{11}=Q_{11} m^{4}+2\left(Q_{12}+2 Q_{66}\right) m^{2} n^{2}+Q_{22} n^{4} \\
& \bar{Q}_{12}=\left(Q_{11}+Q_{22}-4 Q_{66}\right) m^{2} n^{2}+Q_{12}\left(m^{4}+n^{4}\right) \\
& \bar{Q}_{22}=Q_{11} n^{4}+2\left(Q_{12}+2 Q_{66}\right) m^{2} n^{2}+Q_{22} m^{4} \\
& \bar{Q}_{16}=\left(Q_{11}-Q_{12}-2 Q_{66}\right) m^{3} n+\left(Q_{12}-Q_{22}+2 Q_{66}\right) n^{3} m \\
& \bar{Q}_{26}=\left(Q_{11}-Q_{12}-2 Q_{66}\right) m n^{3}+\left(Q_{12}-Q_{22}+2 Q_{66}\right) m^{3} n \\
& \bar{Q}_{66}=\left(Q_{11}+Q_{22}-2 Q_{12}-2 Q_{66}\right) m^{2} n^{2}+Q_{66}\left(m^{4}+n^{4}\right)
\end{aligned}
$$
are:

The stiffness corresponding to transverse deformations

$$
\begin{aligned}
& \bar{Q}_{44}=G_{13} m^{2}+G_{23} n^{2} \\
& \bar{Q}_{45}=\left(G_{13}-G_{23}\right) m n \\
& \bar{Q}_{55}=G_{13} n^{2}+G_{23} m^{2}
\end{aligned}
$$

Where $\mathrm{m}=\cos \theta$ and $\mathrm{n}=\sin \theta$; and $\theta=$ angle between the arbitrary principal axis with the material axis in a layer. The on-axis stiffness is:

\subsection{Strain Displacement Relations}

Green-Lagrange's strain displacement relations are presented in general throughout the analysis. The linear part of the strain is used to derive the elastic stiffness matrix and the non-linear part of the strain is used to derive the geometric stiffness matrix. The total strain is given by

$$
\{\varepsilon\}=\left\{\varepsilon_{l}\right\}+\left\{\varepsilon_{n l}\right\}
$$

The linear generalized shear deformable strain displacement relations are

$$
\begin{gathered}
\varepsilon_{x l}=\frac{\partial u}{\partial x}+\frac{w}{R_{x}}+z k_{x}, \varepsilon_{y l}=\frac{\partial v}{\partial y}+\frac{w}{R_{y}}+z k_{y} \\
\gamma_{x y l}=\frac{\partial u}{\partial y}+\frac{\partial v}{\partial x}+\frac{2 w}{R_{x y}}+z k_{x y}, \gamma_{x z l}=\frac{\partial w}{\partial x}+\theta_{x}-\frac{u}{R_{x}}-\frac{v}{R_{x y}} \\
\gamma_{y z l}=\frac{\partial w}{\partial y}+\theta_{y}-\frac{v}{R_{y}}-\frac{u}{R_{x y}}
\end{gathered}
$$

The bending strains $\mathrm{k}_{\mathrm{j}}$ are expressed as,

$$
\begin{aligned}
& k_{x}=\frac{\partial \theta_{x}}{\partial x}, \quad \mathrm{k}_{\mathrm{y}}=\frac{\partial \theta_{y}}{\partial y} \\
& k_{x y}=\frac{\partial \theta_{x}}{\partial y}+\frac{\partial \theta_{y}}{\partial x}+\frac{1}{2}\left(\frac{1}{R_{y}}-\frac{1}{R_{x}}\right)\left(\frac{\partial v}{\partial x}-\frac{\partial u}{\partial y}\right)
\end{aligned}
$$

The non-linear strain components are as follows:

$$
\begin{aligned}
& \varepsilon_{x n l}=\frac{1}{2}\left(\frac{\partial u}{\partial x}\right)^{2}+\frac{1}{2}\left(\frac{\partial v}{\partial x}\right)^{2}+\frac{1}{2}\left(\frac{\partial w}{\partial x}-\frac{u}{R_{x}}\right)^{2} \\
& +\frac{1}{2} z^{2}\left[\left(\frac{\partial \theta_{x}}{\partial x}\right)^{2}+\left(\frac{\partial \theta_{y}}{\partial x}\right)^{2}\right] \\
& \varepsilon_{y n l}=\frac{1}{2}\left(\frac{\partial u}{\partial y}\right)^{2}+\frac{1}{2}\left(\frac{\partial v}{\partial y}\right)^{2}+\frac{1}{2}\left(\frac{\partial w}{\partial y}-\frac{v}{R_{y}}\right)^{2} \\
& +\frac{1}{2} z^{2}\left[\left(\frac{\partial \theta_{x}}{\partial y}\right)^{2}+\left(\frac{\partial \theta_{y}}{\partial y}\right)^{2}\right]
\end{aligned}
$$




$$
\begin{aligned}
& \gamma_{x y n l}=\frac{\partial u}{\partial x}\left(\frac{\partial u}{\partial y}\right)+\frac{\partial v}{\partial x}\left(\frac{\partial v}{\partial y}\right)+\left(\frac{\partial w}{\partial x}-\frac{u}{R_{x}}\right)\left(\frac{\partial w}{\partial y}-\frac{v}{R_{y}}\right) \\
& +z^{2}\left[\left(\frac{\partial \theta_{x}}{\partial x}\right)\left(\frac{\partial \theta_{x}}{\partial y}\right)+\left(\frac{\partial \theta_{y}}{\partial x}\right)\left(\frac{\partial \theta_{y}}{\partial y}\right)\right]
\end{aligned}
$$

\subsection{Finite Element Formulation}

A Finite element analysis was performed using an eight nodded curved isoparametric element with five degrees of freedom $\mathrm{u}, \mathrm{v}, \mathrm{w}, \theta_{x}$ and $\theta_{y}$ per node. The element is modified to accommodate laminated materials and hygrothermal conditions of the panel, based on first order shear deformation theory where $\mathrm{u}, v$ and $w$ are the displacement components in the $\mathrm{x}, \mathrm{y}, \mathrm{z}$ directions and $\theta_{x}$ and $\theta_{y}$ are the rotations. The stiffness matrix, geometric stiffness matrix due to residual stresses, geometric stiffness matrix due to applied in-plane loads and nodal load vector of the element are derived using the principle of minimum potential energy.

The displacements are expressed in terms of their nodal values by using the element shape functions and are given by.

$$
\begin{gathered}
u(\xi, \eta)=a_{1}+a_{2} \xi+a_{3} \eta+a_{4} \xi^{2} \\
+a_{5} \xi \eta+a_{6} \eta^{2}+a_{7} \xi^{2} \eta+a_{8} \xi \eta^{2} \\
u=\sum_{i=1}^{8} N_{i} u_{i}, v=\sum_{i=1}^{8} N_{i} v_{i}, w=\sum_{i=1}^{8} N_{i} w_{i} \\
\theta_{x}=\sum_{i=1}^{8} N_{i} \theta_{x i}, \theta_{y}=\sum_{i=1}^{8} N_{i} \theta_{y i}
\end{gathered}
$$

$N_{i}=$ Shape function at a node $i$.

\subsection{Stiffness matrix}

The linear strain matrix $\{\varepsilon\}$ is expressed as:

$$
\{\varepsilon\}=[B]\left\{\delta_{e}\right\}
$$

Where $\left\{\delta_{e}\right\}=\left\{u_{1}, v_{1}, w, \theta_{x 1}, \theta_{y 1} \ldots \ldots \ldots, u_{8}, v_{8}, w_{8} \theta_{x 8}, \theta_{y 8}\right\}^{T}$,

$$
[B]=\sum_{i=1}^{8}\left[\begin{array}{ccccc}
N_{i, x} & 0 & 0 & 0 & 0 \\
0 & N_{i, y} & 0 & 0 & 0 \\
N_{i, y} & N_{i, x} & 0 & 0 & 0 \\
0 & 0 & 0 & 0 & N_{i, x} \\
0 & 0 & 0 & N_{i, y} & 0 \\
0 & 0 & 0 & N_{i, x} & N_{i, y} \\
0 & 0 & N_{i, x} & 0 & N_{i} \\
0 & 0 & N_{i, y} & N_{i} & 0
\end{array}\right]
$$

Element stiffness matrix is given by:

$$
\left[K_{e}\right]=\int_{-1}^{+1} \int_{-1}^{+1}[B]^{T}[D][B]|J| d \xi d \eta
$$

2.7. Geometric Stiffness Matrix $\left[K_{G e}^{r}\right]$
The non-linear strain equations are represented in matrix form:

$$
\left\{\varepsilon_{x n l}, \varepsilon_{y n l}, \gamma_{x y n l}, \gamma_{x z n l}, \gamma_{y z n l}\right\}^{T}=[R]\{d\} / 2
$$

Where

$$
\{d\}=\left\{u_{x}, u_{y}, v_{x}, v_{y}, w_{x}, w_{y}, \theta_{x, x}, \theta_{x, y}, \theta_{y, x}, \theta_{y, y}, \theta_{x}, \theta_{y}\right\}^{T}
$$

Equations $\{d\}$ may be expressed as:

$$
\{d\}=\{G\}\left\{\partial_{e}\right\}
$$

$$
\text { Where } \quad[G]=\sum_{i=1}^{8}\left[\begin{array}{ccccc}
N_{i, x} & 0 & 0 & 0 & 0 \\
N_{i, y} & 0 & 0 & 0 & 0 \\
0 & N_{i, x} & 0 & 0 & 0 \\
0 & N_{i, y} & 0 & 0 & 0 \\
0 & 0 & N_{i, x} & 0 & 0 \\
0 & 0 & N_{i, y} & 0 & 0 \\
0 & 0 & 0 & N_{i, x} & 0 \\
0 & 0 & 0 & N_{i, y} & 0 \\
0 & 0 & 0 & 0 & N_{i, x} \\
0 & 0 & 0 & 0 & N_{i, y} \\
0 & 0 & 0 & 1 & 0 \\
0 & 0 & 0 & 0 & 1
\end{array}\right]
$$

The geometric stiffness matrix due to residual stresses is given by:

$$
\left[K_{G e}^{r}\right]=\int_{-1}^{+1} \int_{-1}^{+1}[G]^{T}[S][G]|J| d \xi d \eta
$$

Where

$$
[S]=\left[\begin{array}{ccccccccccccc}
S_{11} & & & & & & & & & & \\
S_{21} & S_{22} & & & & & & & & & \\
0 & 0 & S_{33} & & & & & & & & \\
0 & 0 & S_{43} & S_{44} & & & & & & & \\
0 & 0 & 0 & 0 & S_{55} & & & & & \\
0 & 0 & 0 & 0 & S_{65} & S_{66} & & & & \\
0 & 0 & 0 & S_{73} & S_{74} & 0 & 0 & S_{77} & & & & \\
0 & 0 & S_{83} & S_{84} & 0 & 0 & S_{87} & S_{88} & & & \\
S_{91} & S_{92} & 0 & 0 & 0 & 0 & 0 & 0 & S_{99} & & \\
S_{101} & S_{102} & 0 & 0 & 0 & 0 & 0 & & S_{109} & S_{110} & \\
0 & 0 & S_{113} & S_{114} & 0 & 0 & 0 & 0 & 0 & 0 & 0 & \\
S_{121} & S_{122} & 0 & 0 & 0 & 0 & 0 & 0 & 0 & 0 & 0 & 0
\end{array}\right]
$$

In which

$$
\begin{aligned}
& S_{11}=S_{33}=S_{55}=N_{x}^{r}, S_{22}=S_{44}=S_{66}=N_{y}^{r} \\
& S_{21}=S_{43}=S_{65}=N_{x y}^{r}, S_{77}=S_{99}=N_{x}{ }^{r} t^{2} / 12 \\
& S_{88}=S_{1010}=N_{y}{ }^{r} t^{2} / 12, S_{87}=S_{109}=N^{r}{ }_{x y} t^{2} / 12 \\
& -S_{73}=S_{91}=M_{x}^{r},-S_{84}=S_{102}=M_{y}^{r} \\
& -S_{74}=-S_{83}=S_{92}=S_{101}=M_{x y}^{r} \\
& -S_{113}=S_{121}=Q_{x}^{r},-S_{114}=S_{122}=Q_{y}^{r} \\
& N_{x}^{i r}, N_{y}^{r}, N_{x y}^{r}=\text { in-plane initial internal force }
\end{aligned}
$$
resultants per unit length.

$M^{r}{ }_{x}, M_{y}^{r}, M_{x y}^{r}=$ initial internal moment resultants per unit length. 
$Q_{x}^{r}, Q^{r}=$ initial transverse shear resultants.

\subsection{Geometric Stiffness Matrix $\left[K^{a}{ }_{G e}\right]$}

The first three non-linear strain equations are represented in a matrix form:

$$
\left\{\varepsilon_{x n l}, \varepsilon_{y n l}, \gamma_{x y n l}\right\}^{T}=[U]\{f\} / 2
$$

$\{f\}=\left\{u_{x}, u_{y}, v_{x}, v_{y}, w_{x}, w_{y}, \theta_{x, x}, \theta_{x, y}, \theta_{y, x}, \theta_{y, y}\right\}^{T}$

$\{f\}$ is expressed as:

$$
\{f\}=[H]\left\{\delta_{e}\right\}
$$

Where

$$
[H]=\sum_{i=1}^{8}\left[\begin{array}{ccccc}
N_{i, x} & 0 & 0 & 0 & 0 \\
N_{i, y} & 0 & 0 & 0 & 0 \\
0 & N_{i, x} & 0 & 0 & 0 \\
0 & N_{i, y} & 0 & 0 & 0 \\
0 & 0 & N_{i, x} & 0 & 0 \\
0 & 0 & N_{i, y} & 0 & 0 \\
0 & 0 & 0 & N_{i, x} & 0 \\
0 & 0 & 0 & N_{i, y} & 0 \\
0 & 0 & 0 & 0 & N_{i, x} \\
0 & 0 & 0 & 0 & N_{i, y}
\end{array}\right]
$$

The geometric stiffness matrix due to applied in-plane loads is given by:

$$
\left[K_{G e}^{a}\right]=\int_{-1}^{+1} \int_{-1}^{+1}[H]^{T}[P][H]|J| d \xi d \eta
$$

Where

$$
[P]=\left[\begin{array}{ccccccccccc}
P_{11} & & & & & & & & & \\
P_{21} & P_{22} & & & & & & & & \\
0 & 0 & P_{33} & & & & & & & \\
0 & 0 & 0 & P_{44} & & & & & & \\
0 & 0 & 0 & 0 & P_{55} & & & & & \\
0 & 0 & 0 & 0 & P_{65} & P_{66} & & & & \\
0 & 0 & 0 & 0 & 0 & 0 & P_{77} & & & \\
0 & 0 & 0 & 0 & 0 & 0 & P_{87} & P_{88} & & \\
0 & 0 & 0 & 0 & 0 & 0 & 0 & 0 & P_{99} & \\
0 & 0 & 0 & 0 & 0 & 0 & 0 & 0 & P_{109} & P_{1010}
\end{array}\right]
$$

In which

$$
\begin{aligned}
& P_{11}=P_{33}=P_{55}=N_{x}^{a}, P_{22}=P_{44}=P_{66}=N^{a}{ }_{y} \\
& P_{21}=P_{43}=P_{65}=N_{x y}^{a}, P_{77}=P_{99}=N^{a}{ }_{x} t^{2} / 12 \\
& P_{88}=P_{1010}=N^{a}{ }_{y} t^{2} / 12, P_{87}=P_{109}=N^{a}{ }_{x y} t^{2} / 12 \\
& N_{x}^{a}, N_{y}^{a}, N_{x y}^{a}=\text { applied in-plane forces per unit }
\end{aligned}
$$
length.

\subsection{Load vector}

The element load vector due to the hygrothermal forces and moments is given by:

$$
\left\{P_{e}^{N}\right\}=\int_{-1}^{+1+1} \int_{-1}^{T}[B]\left\{F^{N}\right\}|J| d \xi d \eta
$$

\subsection{Computer Program}

A computer program is developed by using MATLAB environment to perform all the necessary computations. The element elastic stiffness $\left[\mathrm{K}_{\mathrm{e}}\right]$ and mass matrices [M] are derived using a standard procedure. Reduced numerical integration technique by Gaussian quadrature is adopted for the element matrix. Plane stress analysis is carried out using the finite element techniques to determine the stresses and these stresses are used to formulate the geometric stiffness matrix. The overall matrices $[\mathrm{K}],\left[\mathrm{K}_{\mathrm{G}}{ }^{\mathrm{r}}\right],\left[\mathrm{K}_{\mathrm{G}}{ }^{\mathrm{a}}\right]$, and $[\mathrm{M}]$ are obtained by assembling the corresponding element matrices. The boundary conditions are imposed restraining the generalized displacements in different nodes of the discretized structure.

\section{Results and Discussion}

The numerical results of the study of behavior of woven fiber composite plates subjected to hygrothermal loadings are presented using the formulations as follows.

Convergence study

Comparison with previous studies

New results

\subsection{Convergence study}

The convergence study is done for non-dimensional frequencies of free vibration of simply supported square 4 layer symmetric cross-ply and symmetric angle-ply laminated composite plates for elevated temperature and moisture conditions for different mess divisions as shown in Table 1 and Table 2. Convergence is extended to buckling analysis of laminated composite plates subjected to hygrothermal conditions as presented in Table 3 and Table 4 . The study is further extended to dynamic stability analysis of laminated composite plates subjected to hygrothermal conditions as presented in Table 5 and Table 6. As observed, a mesh of $10 \times 10$ shows good convergence of the numerical solution for the free vibration, buckling and dynamic stability of fiber composite plates in hygrothermal environment and this mesh is employed throughout for free vibration, buckling and dynamic stability analysis of woven fiber composite plates in hygrothermal environment.

Table 1. Convergence of non-dimensional fundamental frequencies of free vibration for SSSS four layered laminated composite plates for different ply orientations at 325K temperature

\begin{tabular}{|c|c|c|}
\hline Mess Division & Non- dimensional frequencies at 325K Temperature \\
\hline \multirow{2}{*}{$4 \times 4$} & $0 / 90 / 90 / 0$ & $45 /-45 /-45 / 45$ \\
\cline { 2 - 3 } $6 \times 6$ & 8.079 & 11.380 \\
$8 \times 8$ & 8.039 & 10.785 \\
$10 \times 10$ & 8.036 & 10.680 \\
& 8.036 & 10.680 \\
\hline
\end{tabular}

$\mathrm{a} / \mathrm{b}=1, \mathrm{a} / \mathrm{t}=100$, At $\mathrm{T}=300 \mathrm{~K}, \mathrm{E}_{1}=130 \mathrm{Gpa}, \mathrm{E}_{2}=9.5 \mathrm{Gpa}$, $\mathrm{G}_{12}=6 \mathrm{Gpa}, \mathrm{G}_{13}=\mathrm{G}_{12}, \mathrm{G}_{23}=0.5 \mathrm{G}_{12}, \quad v_{12}=0.3, \quad \alpha_{1}=-0.3 \mathrm{X} 10^{-}$ ${ }^{6} /{ }^{\mathrm{O}} \mathrm{K}, \alpha_{2}=28.1 \mathrm{X} 10^{-6} /{ }^{\mathrm{O}} \mathrm{K}$

Non dimensional frequency, $\lambda=\omega_{\mathrm{n}} \mathrm{a}^{2} \sqrt{\rho / E_{2}} t^{2}$ 
Table 2. Convergence of non-dimensional fundamental frequencies of free vibration for SSSS four layered laminated composite plates for different ply orientations at $\mathbf{0 . 1 \%}$ moisture concentration

\begin{tabular}{|c|c|c|}
\hline $\begin{array}{c}\text { Mess } \\
\text { Division }\end{array}$ & \multicolumn{2}{|c|}{$\begin{array}{c}\text { Non-dimensional frequencies at } 0.1 \% \text { Moisture } \\
\text { concentration }\end{array}$} \\
\hline & $0 / 90 / 90 / 0$ & $45 /-45 /-45 / 45$ \\
$4 \times 4$ & 9.422 & 12.383 \\
$6 \times 6$ & 9.387 & 11.858 \\
$8 \times 8$ & 9.384 & 11.765 \\
$10 \times 10$ & 9.384 & 11.765 \\
\hline
\end{tabular}

$\mathrm{a} / \mathrm{b}=1, \mathrm{a} / \mathrm{t}=100$, At $\mathrm{T}=300 \mathrm{~K}, \mathrm{E}_{1}=130 \mathrm{Gpa}, \mathrm{E}_{2}=9.5 \mathrm{Gpa}$,

$\mathrm{G}_{12}=6 \mathrm{Gpa}, \mathrm{G}_{13}=\mathrm{G}_{12}, \mathrm{G}_{23}=0.5 \mathrm{G}_{12}, v_{12}=0.3 \beta_{1}=0, \beta_{2}=0.44$.

Non dimensional frequency, $\lambda=\omega_{\mathrm{n}} \mathrm{a}^{2} \sqrt{\rho / E_{2}} t^{2}$

Table 3. Convergence of non-dimensional critical load for SSSS four layered laminated composite plates for different ply orientations at 325K temperature

\begin{tabular}{|c|c|c|}
\hline Mess Division & Non- dimensional critical load at 325K Temperature \\
\hline \multirow{3}{*}{$4 \times 4$} & $0 / 90 / 90 / 0$ & $45 /-45 /-45 / 45$ \\
\cline { 2 - 3 } 6 x6 & 0.4481 & 0.6120 \\
$8 \times 8$ & 0.4459 & 0.5818 \\
10x10 & 0.4457 & 0.5764 \\
& 0.4457 & 0.5764 \\
\hline
\end{tabular}

$\mathrm{a} / \mathrm{b}=1, \mathrm{a} / \mathrm{t}=100$, At T=300K, $\mathrm{E}_{1}=130 \mathrm{Gpa}, \mathrm{E}_{2}=9.5 \mathrm{Gpa}$,

$\mathrm{G}_{12}=6 \mathrm{G}$ pa, $\mathrm{G}_{13}=\mathrm{G}_{12}, \mathrm{G}_{23}=0.5 \mathrm{G}_{12}, v_{12}=0.3, \alpha_{1}=-0.3 \quad \mathrm{X} \quad 10^{-}$

${ }^{6} /{ }^{\mathrm{O}} \mathrm{K}, \alpha_{2}=28.1 \mathrm{X} 10^{-6} /{ }^{\mathrm{O}} \mathrm{K}$.

Critical load, $\lambda=\mathrm{N}_{\mathrm{xcr}} /\left(\mathrm{N}_{\mathrm{xcr}}\right)_{\mathrm{C}=0 \%}$ or $\mathrm{T}=300 \mathrm{~K}$

Table 4. Convergence of non-dimensional critical load for SSSS four layered laminated composite plates for different ply orientations at $0.1 \%$ moisture concentration

\begin{tabular}{|c|c|c|}
\hline \multirow{2}{*}{ Mess Division } & \multicolumn{2}{|c|}{$\begin{array}{c}\text { Non- dimensional critical load at } 0.1 \% \text { Moisture } \\
\text { concentration }\end{array}$} \\
\hline \multirow{3}{*}{$4 \times 4$} & $0 / 90 / 90 / 0$ & $45 /-45 /-45 / 45$ \\
6x6 & 0.6095 & 0.7255 \\
$8 \times 8$ & 0.6079 & 0.7041 \\
$10 \times 10$ & 0.6078 & 0.7003 \\
& 0.6078 & 0.7003 \\
\hline
\end{tabular}

$\mathrm{a} / \mathrm{b}=1, \mathrm{a} / \mathrm{t}=100$, At T=300K, $\mathrm{E}_{1}=130 \mathrm{Gpa}, \mathrm{E}_{2}=9.5 \mathrm{Gpa}$,

$\mathrm{G}_{12}=6 \mathrm{Gpa}, \mathrm{G}_{13}=\mathrm{G}_{12}, \mathrm{G}_{23}=0.5 \mathrm{G}_{12}, v_{12}=0.3 \beta_{1}=0, \beta_{2}=0.44$.

Critical load, $\lambda=\mathrm{N}_{\mathrm{xcr}} /\left(\mathrm{N}_{\mathrm{xcr}}\right)_{\mathrm{C}=0 \%}$ or $\mathrm{T}=300 \mathrm{~K}$

Table 5. Convergence of non-dimensional excitation frequency for SSSS four layered laminated composite plates for different ply orientations at 325K temperature

\begin{tabular}{|c|c|c|}
\hline $\begin{array}{c}\text { Mess } \\
\text { Division }\end{array}$ & \multicolumn{2}{|c|}{$\begin{array}{c}\text { Non- dimensional excitation frequency at 325K } \\
\text { Temperature }\end{array}$} \\
\hline & $0 / 90 / 90 / 0$ & $45 /-45 /-45 / 45$ \\
$4 \times 4$ & 15.23 & 22.10 \\
$6 \times 6$ & 15.16 & 20.86 \\
$8 \times 8$ & 15.16 & 20.64 \\
$10 \times 10$ & 15.16 & 20.56 \\
\hline
\end{tabular}

$\mathrm{a} / \mathrm{b}=1, \mathrm{a} / \mathrm{t}=100$, At T=300K, $\mathrm{E}_{1}=130 \mathrm{Gpa}, \mathrm{E}_{2}=9.5 \mathrm{Gpa}$, $\mathrm{G}_{12}=6 \mathrm{Gpa}, \mathrm{G}_{13}=\mathrm{G}_{12}, \mathrm{G}_{23}=0.5 \mathrm{G}_{12}, v_{12}=0.3, \alpha_{1}=-0.3 \times 10^{-}$

${ }^{6} /{ }^{\mathrm{O}} \mathrm{K}, \alpha_{2}=28.1 \times 10^{-6} /{ }^{\mathrm{O}} \mathrm{K}, \alpha=$ static load factor $=0.2, \beta=$ dynamic load factor $=0.2$.

Non-dimensional

excitation

frequency

$$
\left.\Omega=\bar{\Omega} a^{2} \sqrt{\left(\rho / E_{22}\right.} h^{2}\right)
$$

Table 6. Convergence of non-dimensional excitation frequency for SSSS four layered laminated composite plates for different ply orientations at $0.1 \%$ moisture concentration

\begin{tabular}{|c|c|c|}
\hline \multirow{2}{*}{ Mess Division } & \multicolumn{2}{|c|}{$\begin{array}{c}\text { Non- dimensional critical load at } 0.1 \% \text { Moisture } \\
\text { concentration }\end{array}$} \\
\hline & $0 / 90 / 90 / 0$ & $45 /-45 /-45 / 45$ \\
\cline { 2 - 3 } $4 \times 4$ & 18.05 & 24.17 \\
$6 \times 6$ & 17.99 & 23.07 \\
$8 \times 8$ & 17.99 & 22.88 \\
$10 \times 10$ & 17.99 & 22.82 \\
\hline
\end{tabular}

$\mathrm{a} / \mathrm{b}=1, \mathrm{a} / \mathrm{t}=100$, At T=300K, $\mathrm{E}_{1}=130 \mathrm{Gpa}, \mathrm{E}_{2}=9.5 \mathrm{Gpa}$, $\mathrm{G}_{12}=6 \mathrm{Gpa}, \mathrm{G}_{13}=\mathrm{G}_{12}, \mathrm{G}_{23}=0.5 \mathrm{G}_{12}, v_{12}=0.3 \beta_{1}=0, \beta_{2}=0.44, \alpha$ $=$ static load factor $=0.2, \beta=$ dynamic load factor $=0.2$
Non-dimensional

excitation

frequency $\left.\Omega=\bar{\Omega} a^{2} \sqrt{\left(\rho / E_{22}\right.} h^{2}\right)$

\subsection{Comparison with previous studies}

To validate the formulation, the frequencies of vibration the buckling load and excitation frequency during parametric resonance of laminated composite plates are computed and compared with previously published results from literature. The principal instability region of antisymmetric angle-ply composite plates subjected to inplane periodic loads is plotted with non-dimensional frequency $\Omega / \omega$ (ratio of excitation frequency to the free vibration frequency) without static component of load.

\subsection{Free vibration of composite plates subjected to hygrothermal environment}

The present formulation is validated for free vibration analysis of composite plates for temperature and moisture as shown in Table 7 \& Table 8. The four lowest nondimensional frequency parameters due to hygrothermal loadings obtained by the present finite element are compared with numerical solution published by Sairam and Sinha [1] and with those of Shen, Zheng and Huang [3] using finite element method. The present finite element results show good agreement with the previous numerical results published in the literature for free vibration of laminated composite plates subjected to hygrothermal conditions.

Table 7. Comparison of non-dimensional free vibration frequencies for SSSS (0/90/90/0) Plates at 325K temperature

\begin{tabular}{|c|c|c|c|}
\hline \multicolumn{4}{|c|}{ Non- dimensional frequencies at 325K Temperature } \\
\hline $\begin{array}{c}\text { Mode } \\
\text { number }\end{array}$ & $\begin{array}{c}\text { Shen, Zheng and } \\
\text { Huang [3] }\end{array}$ & $\begin{array}{c}\text { Sairam \& Sinha } \\
{[1]}\end{array}$ & $\begin{array}{c}\text { Present } \\
\text { FEM }\end{array}$ \\
\hline 1 & 7.702 & 8.088 & 8.079 \\
\hline 2 & 17.658 & 19.196 & 19.100 \\
\hline 3 & 38.312 & 39.324 & 39.335 \\
\hline 4 & 44.038 & 45.431 & 45.350 \\
\hline
\end{tabular}

$\mathrm{a} / \mathrm{b}=1, \mathrm{a} / \mathrm{t}=100$, At $\mathrm{T}=300 K, \mathrm{E}_{1}=130 \mathrm{Gpa}, \mathrm{E}_{2}=9.5 \mathrm{Gpa}$, $\mathrm{G}_{12}=6 \mathrm{Gpa}, \mathrm{G}_{13}=\mathrm{G}_{12}, \mathrm{G}_{23}=0.5 \mathrm{G}_{12}, v_{12}=0.3, \alpha_{1}=-0.3 \mathrm{X} 10$ ${ }^{6} /{ }^{\mathrm{O}} \mathrm{K}, \alpha_{2}=28.1 \mathrm{X} 10^{-6} /{ }^{\mathrm{O}} \mathrm{K}$.

Non dimensional frequency, $\lambda=\omega_{\mathrm{n}} \mathrm{a}^{2} \sqrt{\rho / E_{2}} t^{2}$

Table 8. Comparison of non-dimensional free vibration frequencies for SSSS (0/90/90/0) Plates at $0.1 \%$ moisture concentration Non- dimensional frequencies at 0.1\% Moisture concentration

\begin{tabular}{|c|c|c|c|}
\hline $\begin{array}{c}\text { Mode } \\
\text { number }\end{array}$ & $\begin{array}{c}\text { Shen, Zheng and } \\
\text { Huang [3] }\end{array}$ & $\begin{array}{c}\text { Sairam \& Sinha } \\
{[1]}\end{array}$ & $\begin{array}{c}\text { Present } \\
\text { FEM }\end{array}$ \\
\hline 1 & 9.413 & 9.429 & 9.422 \\
\hline 2 & 19.867 & 20.679 & 20.597 \\
\hline 3 & 39.277 & 40.068 & 40.084 \\
\hline 4 & 45.518 & 46.752 & 46.708 \\
\hline
\end{tabular}

$\mathrm{a} / \mathrm{b}=1, \mathrm{a} / \mathrm{t}=100$, At $\mathrm{T}=300 \mathrm{~K}, \mathrm{E}_{1}=130 \mathrm{Gpa}, \mathrm{E}_{2}=9.5 \mathrm{Gpa}$, $\mathrm{G}_{12}=6 \mathrm{Gpa}, \mathrm{G}_{13}=\mathrm{G}_{12}, \mathrm{G}_{23}=0.5 \mathrm{G}_{12}, v_{12}=0.3, \beta_{1}=0, \beta_{2}=0.44$.

Non dimensional frequency, $\lambda=\omega_{\mathrm{n}} \mathrm{a}^{2} \sqrt{\rho / E_{2}} t^{2}$

\subsection{Buckling of composite plates subjected to hygrothermal environment}

The present formulation is then validated for buckling analysis of composite plates for elevated temperatures and moistures as shown in Table 9. The square plate has four layers of Graphite / Epoxy composite. The nondimensional critical load due to hygrothermal loadings 
obtained by the present finite element is compared with numerical solution published by Sairam and Sinha [6] and Patel, Ganapathi and Makhecha [24]. The present finite element results show that there exists an excellent agreement with the previously published results in the literature for buckling of composite plates subjected to hygrothermal loads.

Table 9. Comparison of non-dimensional critical load for SSSS $(0 / 90 / 90 / 0)$ four layered laminated composite plates at $325 \mathrm{~K}$ temperature and $0.1 \%$ moisture concentration

\begin{tabular}{|c|c|c|}
\hline References & \multicolumn{2}{|c|}{ Non-dimensional critical load $\lambda$} \\
\hline & At 325K & At $0.1 \%$ \\
\hline Sairam \& Sinha [6] & 0.4488 & 0.6099 \\
Present FEM & $(0.4481)$ & $(0.6095)$ \\
\hline Patel,Ganapathi \& Makhecha [24] & 0.4466 & 0.6084 \\
Present FEM & $(0.4457)$ & $(0.6078)$ \\
\hline
\end{tabular}

$\mathrm{a} / \mathrm{b}=1, \mathrm{a} / \mathrm{t}=100$, At T=300K, $\mathrm{E}_{1}=130 \mathrm{Gpa}, \mathrm{E}_{2}=9.5 \mathrm{Gpa}$, $\mathrm{G}_{12}=6 \mathrm{Gpa}, \mathrm{G}_{13}=\mathrm{G}_{12}, \mathrm{G}_{23}=0.5 \mathrm{G}_{12}, v_{12}=0.3 \alpha_{1}=-0.3 \times 10^{-}$

${ }^{6} /{ }^{\circ} \mathrm{K}, \alpha_{2}=28.1 \times 10^{-6} /{ }^{\circ} \mathrm{K}, \beta_{1}=0, \beta_{2}=0.44$

Non-dimensional Critical load, $\lambda=\mathrm{N}_{\mathrm{xcr}} /\left(\mathrm{N}_{\mathrm{xcr}}\right)_{\mathrm{C}=0 \%}$ or $\mathrm{T}$ $=300 \mathrm{~K}$

\subsection{New results}

After validating the present formulation, the same is employed to study the parametric instability effects of woven fiber composite plates in hygrothermal environment. The geometrical and material properties of the laminated composite plates are: $\mathrm{a}=\mathrm{b}=0.235 \mathrm{~m}$, $\mathrm{h}=0.006 \mathrm{~m}$ (unless otherwise stated). The material properties obtained from tensile testing of glass/epoxy composite plates at different temperatures and moisture are as shown in Table 10 and Table 11.

Table 10. Elastic moduli of glass fiber/epoxy lamina at different temperatures. $\alpha_{1}=-0.3 \times 10^{-6} /{ }^{\circ} \mathrm{K}, \alpha_{2}=28.1 \times 10^{-6} /{ }^{0} \mathrm{~K}, \beta_{1}=0, \beta_{2}=0.44$

\begin{tabular}{|c|}
\hline Temperature in $(\mathrm{K})$ \\
Elastic moduli $300 \mathrm{~K} 325350375400425$ \\
\hline $\mathrm{E}_{1} 7.97 .6 \quad 7.16 .76 .56 .3$ \\
$\mathrm{E}_{2} 7.46 .86 .46 .25 .95 .7$ \\
$\mathrm{G}_{12} 2.92 .62 .32 .11 .81 .6$ \\
$v_{12} 0.40 .430 .410 .350 .360 .35$ \\
\hline
\end{tabular}

Table 11. Elastic moduli of glass fiber / epoxy lamina at different moisture concentrations. $\alpha_{1}=-0.3 \times 10^{-6} /{ }^{\circ} \mathrm{K}, \alpha 2=28.1 \times 10^{-6} /{ }^{\circ} \mathrm{K}, \beta_{1}=0$, $\beta 2=0.44$

\begin{tabular}{|c|}
$\boldsymbol{\beta 2}=\mathbf{0 . 4 4}$ \\
Moisture concentration in \% \\
Elastic moduli 0.0 0.25 0.5 0.75 1.0 \\
\hline $\mathrm{E}_{1} 7.97 .67 .57 .37 .2$ \\
$\mathrm{E}_{2} 7.47 .47 .37 .17 .0$ \\
$\mathrm{G}_{12} 2.92 .92 .82 .72 .6$ \\
$\mathrm{v}_{12} 0.40 .40 .40 .390 .39$ \\
\hline
\end{tabular}

The non-dimensional excitation frequency $\left.\Omega=\bar{\Omega} a^{2} \sqrt{\left(\rho / E_{22}\right.} h^{2}\right)$ is used throughout the dynamic instability studies, where $\bar{\Omega}$ is the excitation frequency in radian/second. The principal instability regions of woven fiber laminated composite flat panel subjected to in-plane periodic loads is plotted with non-dimensional frequency $\Omega / \omega$ (ratio of excitation frequency to the free vibration frequency) versus the dynamic in-plane load $\beta$. Here, a static load factor $\alpha=0.2$ is taken for parametric study of laminated composite plates in hygrothermal environment unless otherwise stated.

The structural instability may lead to large deflection or large amplitude vibrations of structural elements leading to local or global failures. So the analysis is focused on the determination of the primary instability region of laminated composite plates under hygrothermal loads. The width of primary instability region frequencies is the separation of the boundaries of the primary instability region for the given plate. This can be used as an instability measure to study the influence of the other parameters. This is the most dangerous zone and has the greatest practical importance. As can be seen, the primary instability region that occurs in the vicinity of $2 \omega(\alpha=\beta=0)$ and the upper and lower excitation frequencies of the plates decrease with the increase of the static load parameter. It is also observed that the primary instability region for each plate increase with increasing static and dynamic load parameter, and the width of the unstable zone is becoming more significant at the higher load parameter. The spectrum of the values of parameters causing unstable motion is called the dynamic instability region or DIR or parametric resonance. The industry driven woven fiber composite plates subjected to hygrothermal environment are considered here to study the effect of different parameters on the excitation frequency and width of instability regions of composite plates.

The effect of increase in number of layers with thickness of the laminated composite plates on the nondimensional excitation frequency is illustrated in figure 3 at temperature $325 \mathrm{~K}$. The 8,12 and 16 layer laminated plates are having thickness of $6 \mathrm{~mm}, 9 \mathrm{~mm}$ and $12 \mathrm{~mm}$ respectively. As observed, the onset of instability occurs later with narrow instability regions with increase in number of layers due to higher stiffness. As expected, woven fiber laminated plates is more stable with increase in number of layers under periodic loads due to bending stretching coupling.

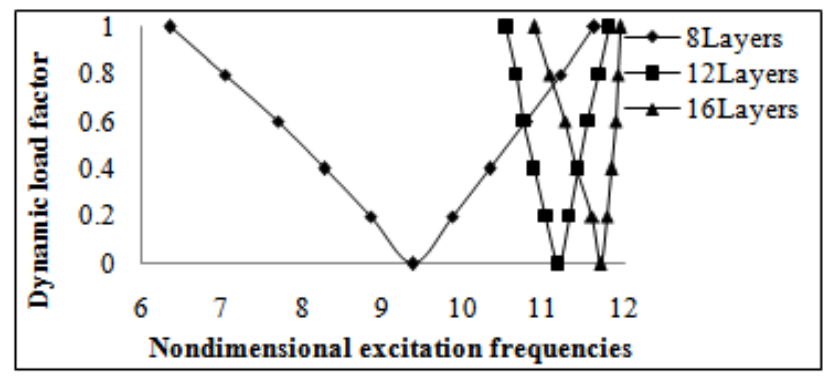

Figure 3. Variation of instability regions with temperature at $325 \mathrm{~K}$ for simply supported (s-s-s-s) of $\mathrm{a} / \mathrm{b}=1, \alpha=0.2$, woven fiber Laminated composite plates

The laminated composite plate affected severely and loses its strength and stiffness in hygrothermal environment. All further parametric studies are done with an eight layers laminate combinations. The effects of number of layers shift the instability region to larger excitation frequencies, which reflect the fact that the instability regions of thicker plates are stiffer. It is observed that the overlapping area of instability regions will become larger when the number of layers increases. The variation of excitation frequency with increase of dynamic load is studied for composite plates with $0.25 \%$ moisture concentration for different increase in number of layers as shown in figure 4. As observed, the onset of instability occurs earlier and the width of instability regions becomes smaller with decrease in number of layers. 


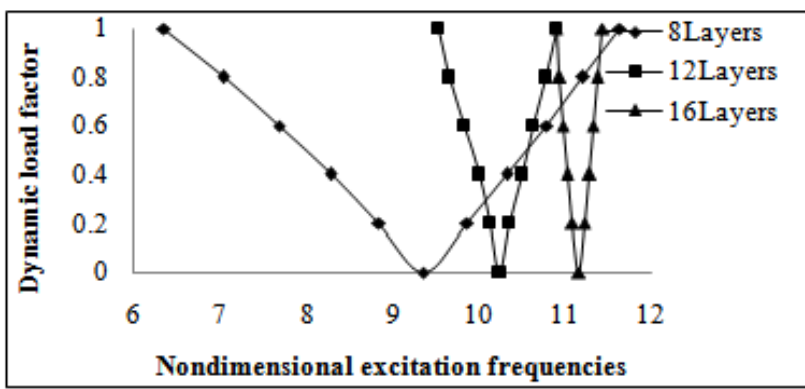

Figure 4. Variation of instability regions with moisture concentration at $0.25 \%$ for simply supported (s-s-s-s) of $\mathrm{a} / \mathrm{b}=1, \alpha=0.2$, woven fiber Laminated composite plates

The effect of increase in aspect ratio for $\mathrm{a} / \mathrm{b}=0.5,1$ and 2 on the non-dimensional excitation frequencies is analyzed for composite plates as shown in figure 5 at temperature $325 \mathrm{~K}$. The onset of instability occurs earlier for composite plates with increase in aspect ratio. With increase in aspect ratios the excitation frequencies are decreased, due to reduction of effective stiffness of the plates in hygrothermal environment.

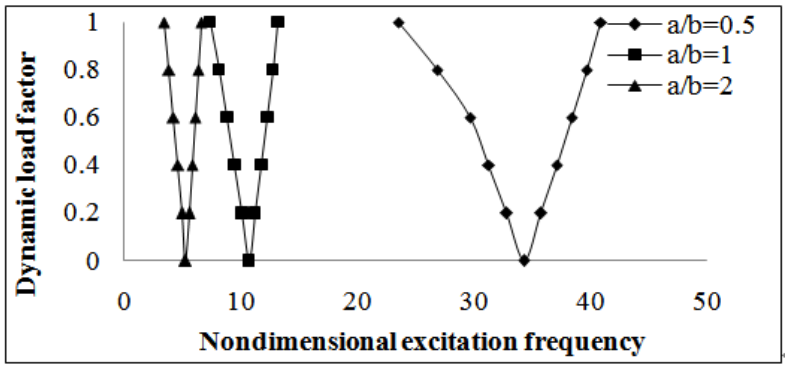

Figure 5. Variation of instability regions with temperature at 325K for simply supported (s-s-s-s) of $\mathrm{a} / \mathrm{b}=1, \alpha=0.2$, woven fiber Laminated composite plates

The effect of increase in aspect ratios on the nondimensional excitation frequencies is studied for composite plates as shown in figure 6 at moisture concentration $0.25 \%$. The onset instability occurs later for square plates than rectangular plates with wider instability region. The excitation frequencies are decreased with increase in aspect ratios in hygrothermal environment.

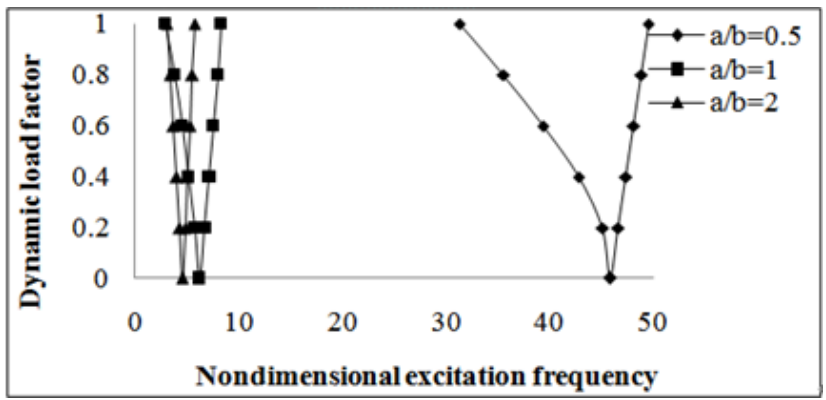

Figure 6. Variation of instability regions with moisture concentration at $0.25 \%$ for simply supported (s-s-s-s) of $\mathrm{a} / \mathrm{b}=1, \alpha=0.2$, woven fiber Laminated composite plates

The effect of increase in side to thickness ratio for $\mathrm{b} / \mathrm{t}=25$ and 50 on the non-dimensional excitation frequencies is investigated for composite plates as shown in figure 7 at temperature $325 \mathrm{~K}$. It is observed from the figure that the onset of instability occurs earlier for increase in side to thickness ratio. The excitation frequencies are decreased with increase in side to thickness ratio. The thick plates are more dynamically stable than thin plates in temperature environment.

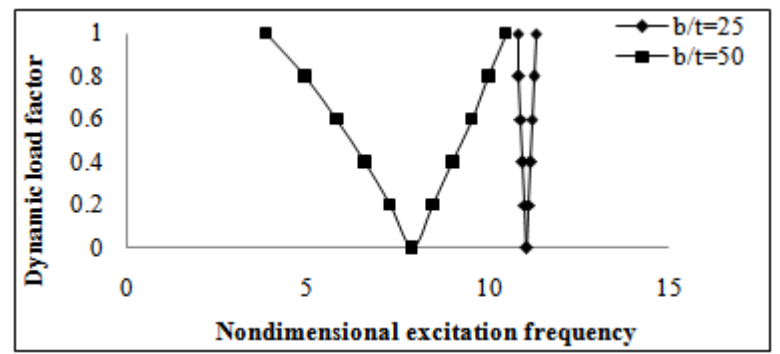

Figure 7. Variation of instability regions with temperature at $325 \mathrm{~K}$ for simply supported (s-s-s-s) of $\mathrm{a} / \mathrm{b}=1, \alpha=0.2$, woven fiber Laminated composite plates

The effect of increase in side to thickness ratio for $\mathrm{b} / \mathrm{t}=25$ and 50 on the non-dimensional excitation frequencies is investigated for composite plates as shown in figure 8 at a moisture $0.25 \%$. It is observed from the figure that the onset of instability occurs earlier for increase in side to thickness ratio. The excitation frequencies are decreased with increase in side to thickness ratio. The thick plates having narrow instability region shows more stiffness and strength than thin plates in hygrothermal environment. The width of instability regions increases due to increase in dynamic loads.

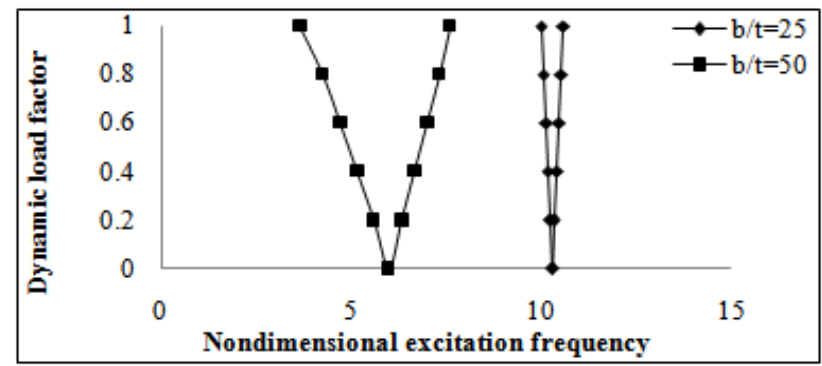

Figure 8. Variation of instability regions with moisture concentration at $0.25 \%$ for simply supported (s-s-s-s) of $\mathrm{a} / \mathrm{b}=1, \alpha=0.2$, woven fiber Laminated composite plates

The effect of increase in static load factor of eight layered anti-symmetric woven fiber laminated composite plates on non-dimensional excitation frequency is analyzed in figure 9 at temperature $325 \mathrm{~K}$.

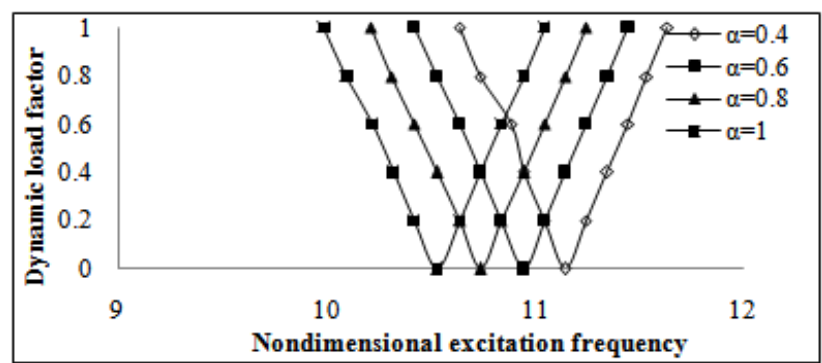

Figure 9. Variation of instability regions with temperature at $325 \mathrm{~K}$ for simply supported (s-s-s-s) of $[45 /-45]_{4}$, woven fiber Laminated composite plates

It is observed that with the increase of static load factor from 0.4 to 1 , the onset of dynamic instability occurs earlier and the width of dynamic instability region also increases. The reduction of excitation frequencies with increase in static load factors from 0.4 to 1 is more than $10 \%$ 
due to reduction of stiffness and strength. All further studies are done with a static load factor $\alpha=0.2$.

The variations of dynamic instability regions with increase in static in-plane loads of woven fiber composite plates at moisture concentrations $0.25 \%$ is observed as shown in figure 10 . The onset of instability occurs earlier with increase of compressive static in-plane load, the instability region tend to shift to lower frequencies and wider. The reduction of excitation frequencies with increase in static in-plane loads from 0.4 to 1 is more than $5 \%$.

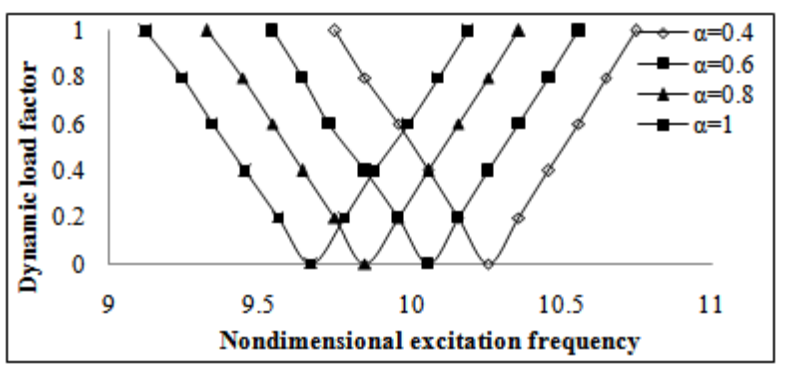

Figure 10. Variation of instability regions with moisture concentration at $0.25 \%$ for simply supported (s-s-s-s) of [45/-45 $]_{4}$, woven fiber Laminated composite plates

The variations of dynamic instability of composite plates with increase in temperature from $325 \mathrm{~K}$ to $400 \mathrm{~K}$ is shown in figure 11 with a static load factor 0.2 , it is observed that the onset of instability occurs earlier with increase in temperature. The width of instability region decreased with increase in temperature from $325 \mathrm{~K}$ to $400 \mathrm{~K}$. The reduction of excitation frequencies with increase in temperature from $325 \mathrm{~K}$ to $400 \mathrm{~K}$ is about $50 \%$.

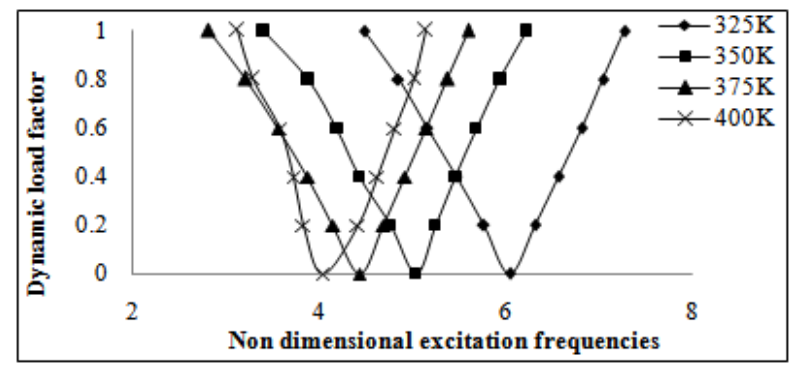

Figure 11. Variation of instability regions with different temperature for simply supported (s-s-s-s) of $[45 /-45]_{4}$, woven fiber Laminated composite plates

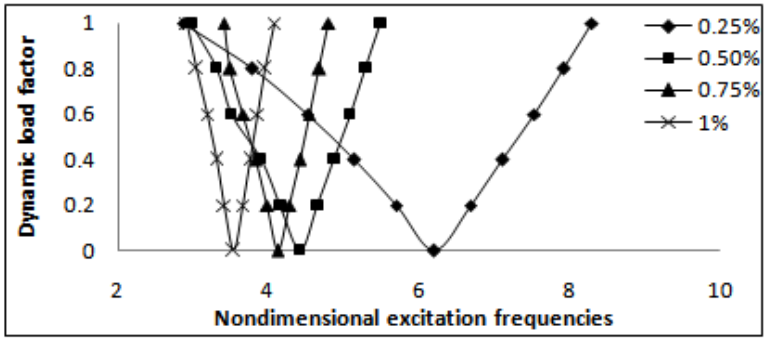

Figure 12. Variation of instability regions with different moisture concentration for simply supported (s-s-s-s) of $[45 /-45]_{4}$, woven fiber Laminated composite plates

Similarly with increase in moisture concentration from $0.25 \%$ to $1 \%$ is shown in figure 12 with static load factor 0.2 , it is observed that the onset of instability occurs earlier with increase in moisture concentration. The width of instability region decreased with increase in moisture concentration from $0.25 \%$ to $1 \%$. The reduction of excitation frequencies with increase in moisture concentration from $0.25 \%$ to $1 \%$ is more than $50 \%$.

The study is further extended to study the effect of different laminated parameter on composite plates under high temperature environment, here considered at temperature $325 \mathrm{~K}$. As shown in figure 13, it is observed that the onset of instability occurs for symmetric laminates earlier than anti-symmetric laminates. The nondimensional excitation frequencies decreased $50 \%$ for laminates with symmetric lay-up than anti-symmetric layup in temperature environment.

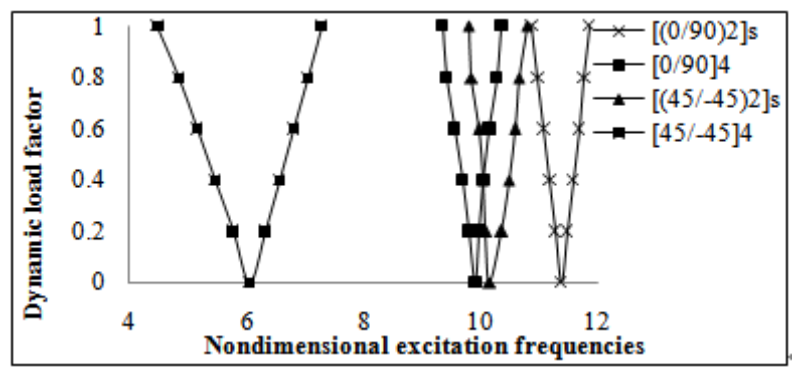

Figure 13. Variation of instability regions with temperature at $325 \mathrm{~K}$ for simply supported (s-s-s-s) $\alpha=0.2$, woven fiber Laminated composite plates

A study is also under taken to study the effect of different lamination sequence on instability of composite plates under moisture environment. Similar symmetric and anti-symmetric laminates having different lamination sequence with moisture concentration $0.25 \%$ are considered in figure 14.

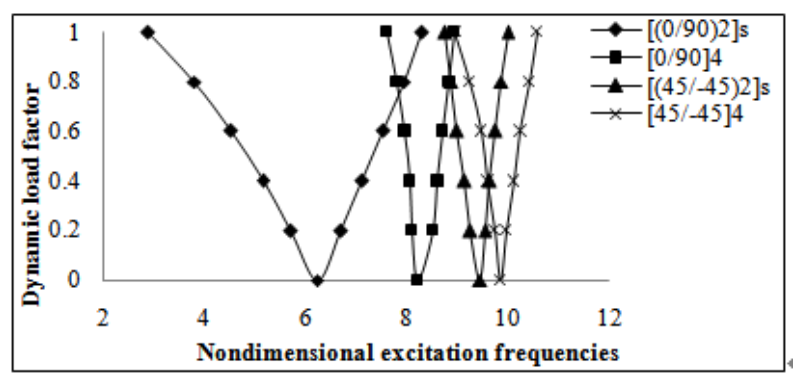

Figure 14. Variation of instability regions with moisture concentration at $0.25 \%$ for simply supported (s-s-s-s), $\alpha=0.2$, woven fiber Laminated composite plates

The onset of instability occurs earlier for symmetric laminates with wider instability regions, where as the narrow instability regions for anti-symmetric laminates. Plates with $45^{\circ}$ ply-orientations seem to be stiffer due to shifting of higher frequencies and narrow instability region. The non-dimensional excitation frequencies decreased $50 \%$ for laminates with symmetric lay-up than anti-symmetric lay-up in moisture environment.

Further it is noticed, in most of the cases, in which the dynamic instability regions overlap due to the amplitude of the static and dynamic in-plane load $\alpha$ and $\beta$ increases in hygrothermal environment. The width of instability region increases with increase in both static and dynamic load parameters. Furthermore, for a higher load, the antisymmetric angle-ply woven fiber laminated plate is unstable for a wide range of excitation frequencies, due to the overlapping of different instability regions associated with higher frequencies in hygrothermal environment. 
Further studies are done with anti-symmetric angle-ply laminated plates.

The effect of ply-orientation $0^{\circ}$ to $90^{\circ}$ on the nondimensional excitation frequencies is reported for antisymmetric angle-ply laminated plates at temperature 325K as shown in figure 15.

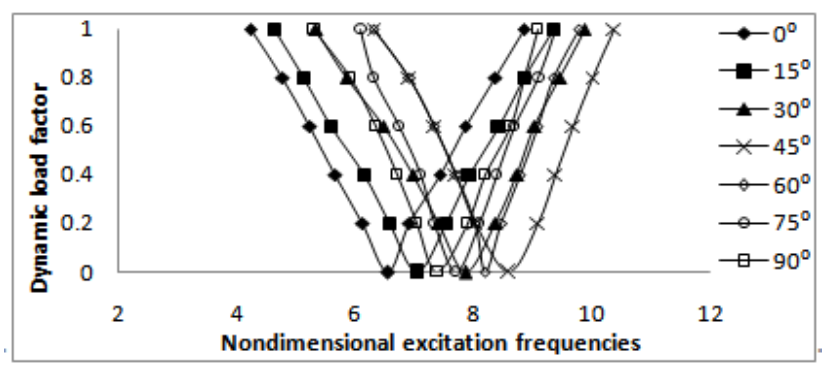

Figure 15. Variation of instability regions with temperature at $325 \mathrm{~K}$ for simply supported (s-s-s-s) of [45/-45 $]_{4}, \alpha=0.2$, woven fiber Laminated composite plates

The onset of instability occurs earlier with decrease of lamination angle. The excitation frequencies beyond lamination angle $45^{\circ}$ decreased marginally. The excitation frequencies are decreased with increase in lamination angle due to reduction of stiffness and strength of laminated plates. The reduction of excitation frequencies with increase in lamination angle from $0^{\circ}$ to $45^{\circ}$ is about $25 \%$. The onset of instability and the width of instability region and its strength is highly depends on lamination angle. The greater the lamination angle the smaller is the width of instability region. The rectangular laminated plates show that the lamination angle of $45^{\circ}$ is symmetrical. For this the lamination angle of $45^{\circ}$ seems to be the preferential ply orientation for the lamination sequence which is due to the dominance effect of bendingstretching coupling of laminates.

The effects of increase in lamination angle $0^{\circ}$ to $90^{\circ}$ on the non-dimensional excitation frequencies is reported for composite plates at moisture concentration $0.25 \%$ is shown in figure 16. It is observed from the figure that the onset of instability occurs for lower values of lamination angle. The reduction of excitation frequencies with increase in lamination angle $0^{\circ}$ to $45^{\circ}$ is about $50 \%$. Which shows that the anti-symmetric angle-ply laminated plates is severely affected and reduced its maximum strength and stiffness with increase in lamination angle in moisture environment.

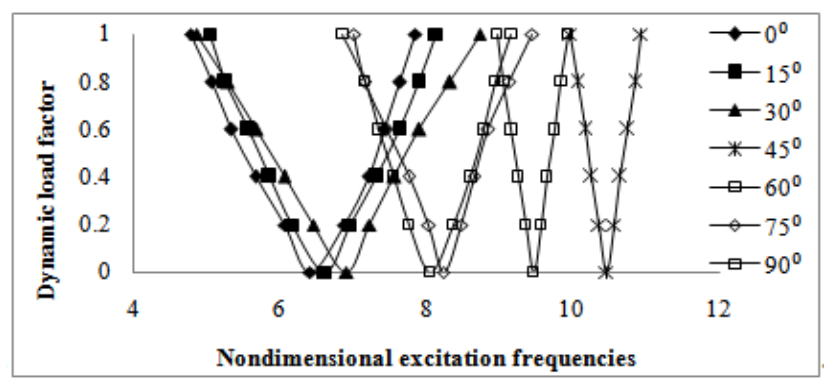

Figure 16. Variation of instability regions with moisture concentration at $0.25 \%$ for simply supported (s-s-s-s), $\alpha=0.2$, woven fiber Laminated composite plates

The effect of degree of orthotropy is examined for the eight layered anti-symmetric angle-ply laminated plates on the non-dimensional excitation frequency as shown in figure 17 at temperature $325 \mathrm{~K}$. It is seen that with increase in the values of $E_{1} / E_{2}=10,20$ and 40 , the onset of instability occurs earlier with decrease in degree of orthotropy. The width of instability zones decreases with increase of degree of orthotropy in temperature environment.

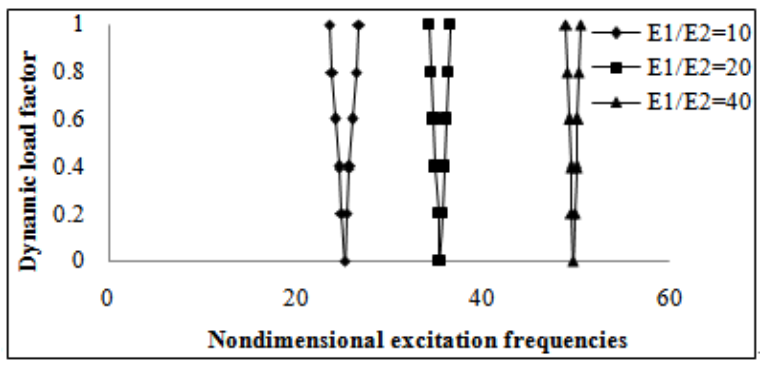

Figure 17. Variation of instability regions with temperature at $325 \mathrm{~K}$ for simply supported (s-s-s-s) of [45/-45 $]_{4}, \alpha=0.2$, woven fiber Laminated composite plates

With the increase of degree orthotropy i.e. the increase in $E_{1} / E_{2}$ the instability occurs at higher frequencies. The reduction of excitation frequency with increase in $E_{1} / E_{2}$ $=10$ to 40 about $50 \%$. This shows that there is worst variation of stiffness and strength with increase in degree of orthotropy characteristics.

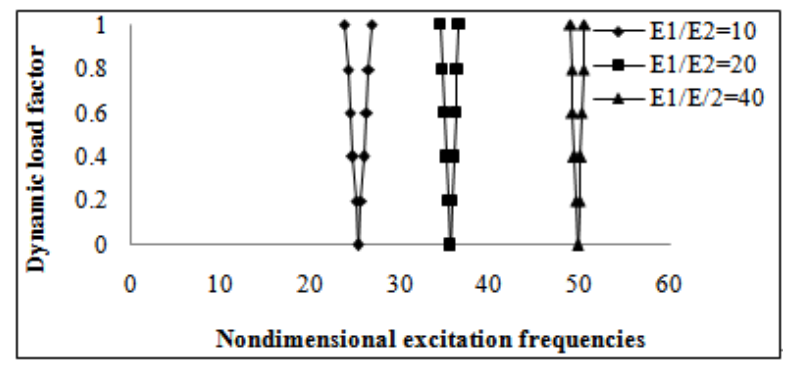

Figure 18. Variation of instability regions with moisture concentration at $0.25 \%$ for simply supported of $[45 /-45]_{4}, \alpha=0.2$, (s-s-s-s) woven fiber Laminated composite plates

The variations of dynamic instability regions with increase in the values of degree of orthotropy $E_{1} / E_{2}=10$, 20 and 40 are presented as shown in figure 18 at moisture concentration $0.25 \%$. The onset of instability occurs earlier with decrease in degree of orthotropy. The degree of instability is narrow with increase in degree of orthotropy of laminated plates, which shows more stiffness and strength in hygrothermal environment.

The reduction of excitation frequency is about to $50 \%$ with increase in degree of orthotropy $E_{1} / E_{2}=10$ to 40 . This shows that the isotropic and orthotropic laminated plates are least deterioration of stiffness and strength in extreme hygrothermal environment. The larger the ratio of $E_{1} / E_{2}$, for the high-modulus-fiber material plates, the laminated plates is most stable and better stiffness in adverse hygrothermal environment.

\section{Conclusions}

The parametric instability study of woven fiber laminated composite plates in hygrothermal environment subjected to periodic in- plane loads is examined. A general formulation for dynamic instability of laminated composite plates subjected to hygrothermal loadings is 
presented for the first time. From the detailed study, the following observation can be made:

The excitation frequencies of laminated fiber composite plates decrease with increase of temperature and moisture concentration due to reduction of stiffness for all laminates. The greater is the lamination angle the smaller is the instability region of composite plates in hygrothermal environment.

With increase in aspect ratios the excitation frequencies are decreased, due to reduction of effective stiffness of the plates in hygrothermal environment.

Woven fiber laminated plates is more stable with increase in number of layers in hygrothermal environment

The thick plates having narrow instability region shows more stiffness and strength than thin plates in hygrothermal environment.

The onset of instability occurs for symmetric laminates earlier than anti-symmetric laminates in hygrothermal environment.

The width of instability zones decreases with increase of degree of orthotropy in hygrothermal environment.

The high-modulus-fiber material plate is most stable in hygrothermal environment.

The width of the instability regions increases with increase in both static and dynamic load parameters.

Instability occurs earlier with an increase of the static compressive in-plane load, the instability regions tend to shift to lower frequencies, showing a destabilizing effect on the dynamic stability behavior of the plates in hygrothermal environment.

From the present studies, it is concluded that the parametric instability behavior laminated plates is greatly influenced by the geometry, aspect ratio, side to thickness ratio, static load factor and degree of orthotroy. Such a property can be utilized to tailor the design of woven fiber laminated composite plates in hygrothermal environment.

\section{References}

[1] KS Sai Ram and P.K. Sinha, Hygrothermal effects on the free vibration of laminated composite plates, J. of. Sound. and Vibr. 158 (1992) 133-148.

[2] B. Chen, and T- W. Chou, Free vibration analysis of orthogonalwoven fabric composites, J. of. Compo. Parts A. 30 (1999) 285297.

[3] H-S. Shen, J-J. Zheng and X-L.Huang, The effects of Hygrothermal conditions on the dynamic response of shear deformable laminated plates resting on elastic foundations, J. of Reinf. Plast. and Compo. 23 (2004) 1095-1113.

[4] V. Fakhari and A. Ohadi, Nonlinear vibration control of functionally graded plate with piezoelectric layers in thermal environment. J. of Vib.and Cont. (3) (2011) 449-469.
[5] B R. Dewey and G A. Costello, Thermal buckling of nonhomogeneous plates, Nucl. Engg. and Design. 7 (1968) 249261.

[6] K S. Sai Ram and P K.Sinha, Hygrothermal effects on the buckling of laminated composite plates, J. of Compo. Struct. 21 (1992) 233-247.

[7] R M. Jones, Thermal buckling of uniformly heated unidirectional and symmetric cross-ply laminated fiber-reinforced composite uniaxial in-plane restrained simply supported rectangular plates, J. of Compo. Part A. 36 (2005) 1355-1367.

[8] P.K. Dash, R. Sathisbabu, and C. Ganesan, Effect of corrosive environment on elasto-buckling strength of GFRC Plate. Asian. J. of Mater. Sci. (2011) 1-15.

[9] C W. Bert and V. Birman, Dynamic instability of shear deformable anti-symmetric angle-ply plates, Inter. J. Solids. Struct. 23 (1987) 1053-1067.

[10] J. Moorty J N Reddy and RH Plaut, Parametric instability of laminated composite plates with transverse shear deformation. Inter. J. Solids. Struct. 26 (1990) 801-811.

[11] Z. Cheng-ti, Theory of nonlinear dynamic stability for composite laminated plates, J. of Appl. Mathe. and Mecha. 12 (1991) 113120.

[12] M. Mond and G. Cederbaum, Dynamic instability of antisymmetric laminated plates, J. of Soun. And Vibr. 154 (1992) 271-279.

[13] C-Li. Liao and C-R. Cheng, Dynamic stability of stiffened laminated composite plates and shells subjected to in-plane pulsating forces, Int. J. for Num. Method. in Engin. 37 (1994) 4167-4183.

[14] M. Ganapathi, Dynamic instability of laminates subjected to temperature field, J. of Engin. Mecha. 124 (1998) 1166-1168.

[15] A. Chatopadhyay and A G. Radu, Dynamic instability of composite laminates, J. of Compu. and Struct. 77 (2000) 453-460.

[16] S. K. Sahu and P. K. Datta, Dynamic instability of laminated composite rectangular plates subjected to non- uniform harmonic in-plane edge loading, J. of Aero. Engg. 214 (2000) 295-312.

[17] S. Wang and D J Dawe, Dynamic instability of composite laminated rectangular plates and prismatic Plate structures. J. of Compu. Method. in Appl. Mecha. and Engg. 191 (2002) 17911826.

[18] G-Y. Wu and Y-S.Shih, Dynamic stability of rectangular plate with an edge crack, J of Compu. and Struc. 84 (2005) 1-10.

[19] G Y. Wu and Y S. Shih, Analysis of dynamic instability for arbitrarily laminated skew plates, J. of Sou. and Vibr. 292 (2006) 315-340.

[20] K M. Liew, Y Y. Lee, T Y Ng and X. Zhao, Dynamic stability analysis of composite cylindrical panels, Int. J. of Mecha.Scien. 49 (2007) 1156-1165.

[21] Wu Lanhe, W. Hangjur and Daobin, Dynamic stability analysis of functionally graded material plates by the moving square differential quadrature method, J. of Comp. Struct. 77 (2007) 383394.

[22] Chen. Chun-Sheng, Chen Wei-Ren and Chien, Rean-Der, Stability parametric vibrations of hybrid Composite plates, Euro. J. of Mech. A/Solids. 28 (2009) 329-337.

[23] J. Fazilati and H.R. Ovesy, Dynamic instability analysis of composite laminated thin walled structures using two version of FSM. J. of Comp. Struc. 92 (2010) 2060-2065.

[24] B.P. Patel, M. Ganapathi and D. P.Makhecha, Hygrothermal effects on the structural behavior of thick Composites using higher-order theory Compo. Structs. 56 (2002) 25-34. 